$16^{\text {th }}$ International Congress of Metrology, 10008 (2013)

DOI: $10.1051 /$ metrology/201310008

(C) Owned by the authors, published by EDP Sciences, 2013

\title{
Establishment of metrological traceability in chemistry: an overview of two INRiM activities
}

\author{
Elena Orrù ${ }^{1, a}$ and Francesca Rolle ${ }^{2}$ \\ ${ }^{1}$ Istituto Nazionale di Ricerca Metrologica (INRiM), Electromagnetism Division, Strada delle Cacce 91, I-10135 Torino, \\ Italy \\ ${ }^{2}$ Istituto Nazionale di Ricerca Metrologica (INRiM), Thermodynamics Division, Strada delle Cacce 91, I-10135 Torino, \\ Italy
}

\begin{abstract}
Quality control is fundamental in many fields as environmental monitoring, industrial processes and food safety. It is necessary to use reliable methods and instrumentation to perform accurate and efficient measurements, to prevent possible adverse effects on human health and to optimise the industrial final products. The contribution of metrology is fundamental, as it provides the means to obtain accurate, traceable measurement results, which can be compared even if determined in different places and times. In this work we present two activities concerning metrology in chemistry carried out at Istituto Nazionale di Ricerca Metrologica (INRiM), the Italian National Metrology Institute.
\end{abstract}

\begin{abstract}
Le contrôle qualité est fondamental dans de nombreux domaines tels que la surveillance de l'environnement, les procédés industriels et de la sécurité alimentaire. Il est nécessaire d'utiliser des méthodes et d'instruments fiables pour effectuer des mesures exactes et efficace, afin d'éviter les possibles effets négatifs sur la santé humaine et à optimiser les produits finis industriels. La contribution de la métrologie est fondamentale, car elle fournit les moyens pour obtenir des résultats de mesure exacts et traçables, qui peuvent être comparés, même s'ils sont déterminés à différents endroits et moments. Dans ce travail, nous présentons deux activités relatives à la métrologie en chimie effectuées à l'Istituto Nazionale di Ricerca Metrologica (INRiM), l'Institut National de Métrologie Italien.
\end{abstract}

\section{Organic micropollutants determination in real matrices}

\subsection{Introduction}

INRiM activity concerning the determination of organic micropollutants at trace concentrations in various matrices (food, environmental samples), aims at establishing metrological traceability to the International System of Units (SI) and correctly evaluating the measurement uncertainty for the results of these measurements. The possibility of analysing these substances with accurate and metrologically traceable methods is a goal of fundamental importance, both in the food safety and the environmental fields, due to the

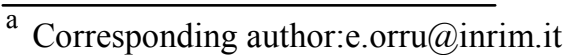

potential toxic effects that these substances can act on human health and on natural ecosystems. The classes of compounds considered until now are Polychlorobyphenils (PCBs), Polycyclic Aromatic Hydrocarbons (PAHs) and some organochlorine pesticides classified as Persistent Organic Pollutants (POPs) by the United Nation Environment Programme [1], in the framework of the Stockholm Convention.

The activity consists in the development and validation of suitable analytical methods in order to establish correct metrological traceability chains. The development of such traceability chains cannot be easy, due to several problems: presence of a great amount of analytes in the samples at various concentrations (even at trace levels), complexity of the environmental samples and matrix effects, lack of primary methods, applicable to 
routine measurements, which allow the direct reference to a measurement standard. A fundamental aspect regards the evaluation of measurement uncertainty considering all the significant contributions.

\subsection{Analysis of organochlorine pesticides in tea}

We started an activity regarding the analysis of some organochlorine pesticides (OCPs) in food matrices, in the framework of the participation in the CCQM Pilot Study P136 "Mid-Polarity Analytes in Food Matrix: MidPolarity Pesticides in Tea", which concerned the determination of the mass fractions of two pesticides, $\beta$ endosulfan and its metabolite endosulfan sulfate in a food matrix, i.e. green tea powder, between $100 \mu \mathrm{g} / \mathrm{kg}$ to 1000 $\mu \mathrm{g} / \mathrm{kg}$. Endosulfan is a broad-spectrum insecticide, widely used in agricultural practices, which was banned in 2011 as it is a strong neurotoxic agent, both on insects and on mammals, including humans. In addition endosulfan is an endocrine disruptor (agent that can "mime" the activity of some hormones) and many studies have documented its reproductive and developmental toxicity. Endosulfan can also bioaccumulate in the food chain, displaying high toxicity [2]. All these features supported its inclusion in the POPs classification in 2009. The CCQM-P136 pilot study (and the parallel Key Comparison CCQM-K95), coorganised by Government Laboratory of Hong Kong (GLHK - Hong Kong) and National Institute of Metrology (NIM - China), required the development of a procedure which involved extraction, clean-up, analytical separation and selective detection of the analytes in the food matrix. The samples of green tea object of the comparison were prepared by GLHK starting from a batch of Chinese commercial tea purchased from a local market. Two bottles of sample were sent to the participants, one for the method development and another for the analysis with the preferred method. The comparison was designed to test the capabilities of the participant National Metrology Institutes (NMIs) for determining mid-polarity analytes in a food matrix.

\subsection{Method development}

For the method development, the following steps had to be carried out:

- set up of the analytical procedure for the extraction of the pesticides from the matrix and preparation of the samples for the quantification step (clean-up, concentration);

- set up of the best analytical conditions for the quantification by means of gas-chromatography coupled with mass spectrometry (GS-MS);

- evaluation of measurement uncertainty.

The metrological traceability was established using suitable Certified Reference Materials (CRMs), both for the calibration of the analytical instrumentation and for the evaluation of the recovery efficiency of the analytes from the matrix. For the calibration of the GC-MS a
CRM produced by the National Institute of Standards and Technology (NIST) was used (i.e. NIST SRM 2275) which consists of an organic solution containing some OCPs, among which endosulfan and endosulfan sulfate. The calibration solutions were prepared by gravimetric dilution of the CRM, in order to have different concentrations of the analytes in the range of interest.

The calibration curves were obtained by means of an algorithm based on the Weighted Least Squares, developed at INRiM [3], which calculates a linear correction to be applied to the instrument readings of the calibration solutions. This correction can be applied to the instrumental readings of the unknown samples, thus obtaining the correct values. The algorithm takes into account different sources of uncertainty, the standard solutions uncertainty, the repeatability of the instrument, the lack of fit, the instrumental resolution. The model equation used to determine the mass fractions $\chi_{\mathrm{a}}$ of the pesticides is the following (eq. 1):

$$
\chi_{\mathrm{a}}=\frac{c_{\mathrm{a}} \cdot V_{\mathrm{E}}}{R \cdot m_{\mathrm{h}} \cdot f_{\mathrm{h}}}
$$

where:

$\chi_{\mathrm{a}}:$ mass fraction of each analyte in $\mu \mathrm{g} / \mathrm{kg}$

$c_{\mathrm{a}}$ : is the analyte concentration in $\mu \mathrm{g} / \mathrm{mL}$ determined in the sample extract by GC-MS quantification

$V_{\mathrm{E}}$ : is the final volume of the extract in $\mathrm{mL}$, determined by weighing

$R:$ is the recovery factor

$m_{\mathrm{h}}$ : is the weighed mass of the sample in $\mathrm{kg}$

$f_{\mathrm{h}}$ : is the correction factor for moisture content.

The recovery efficiency $R$ was determined by spiking samples of commercial green tea with known amounts of the two pesticides, then processing and analysing these samples as the comparison samples to take into account the possible losses during the sample preparation procedure.

$R$ was calculated from eq. 2 , which is the ratio of the measured concentrations $\chi_{\text {calc }}$ and the theoretical concentrations in the spiked samples $\chi_{\text {theor }}$ :

$$
R=\frac{\chi_{\text {calc }}}{\chi_{\text {theor }}}
$$

The evaluation of the moisture content of the sample was performed in order to determine the mass fractions of the pesticides on a dry mass basis, as requested by the protocol of the comparison. The moisture content of green tea samples was determined by weighing some aliquots of tea before and after heating them to constant weight, thus obtaining by difference the content of humidity in the samples.

The developed method is summarised in fig. 1: 


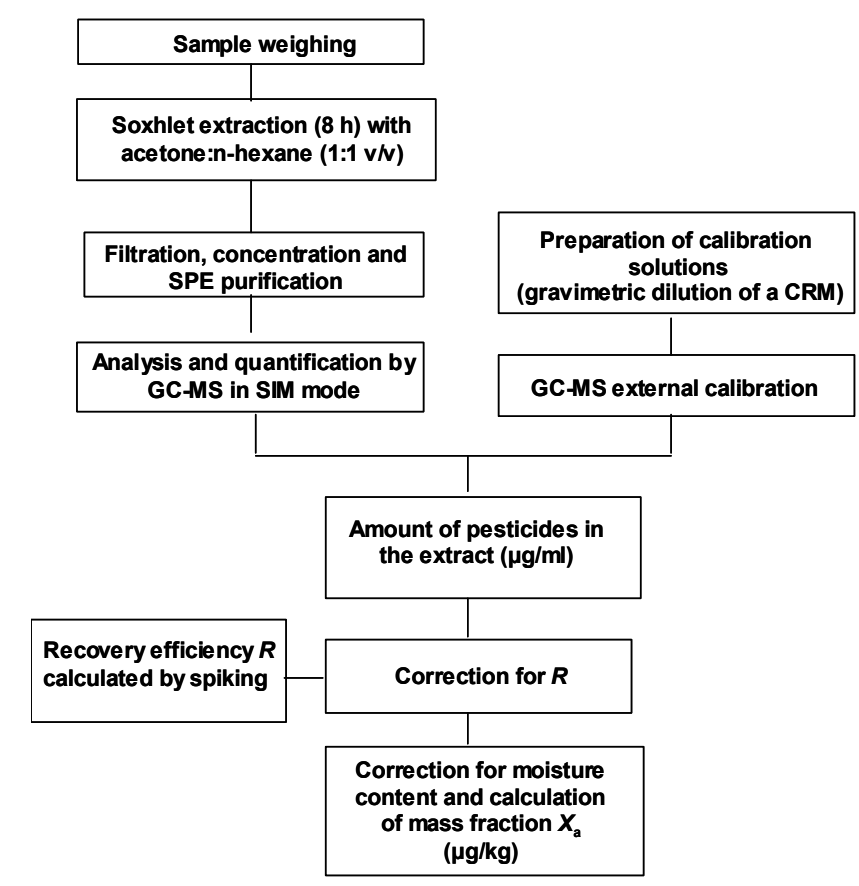

Figure 1. Method for the quantification of $\beta$-endosulfan and endosulfan sulfate in tea.

\subsection{Uncertainty evaluation}

We carried out the evaluation of measurement uncertainty starting from the model equation (1), taking into account all the significant sources for each input quantity. In table 1 , an example of uncertainty budget for $\beta$-endosulfan is shown. The major contributions to the combined standard uncertainty $u_{\mathrm{c}}\left(\chi_{\mathrm{a}}\right)$ are related to the analyte concentration determined by GC-MS $u\left(c_{\mathrm{a}}\right)$ and the recovery efficiency $u(R)$.

Table 1. Example of uncertainty budget for $\beta$-endosulfan

\begin{tabular}{|c|c|c|c|c|c|}
\hline $\begin{array}{c}\text { Input } \\
\text { quantity } \\
x_{i}\end{array}$ & $\begin{array}{c}\text { Uncerta } \\
\text { inty } \\
\text { compon } \\
\text { ent } \\
u\left(x_{i}\right)\end{array}$ & $\begin{array}{l}\text { Uncertainty } \\
\text { source }\end{array}$ & $\begin{array}{c}\text { Input } \\
\text { quantity } \\
\text { value } \\
x_{i}\end{array}$ & $\begin{array}{c}\text { Standard } \\
\text { uncertainty } \\
\text { value } \\
u\left(x_{i}\right)\end{array}$ & $\begin{array}{c}\text { Contribution } \\
\text { to } u_{c}\left(\chi_{a}\right) / \chi_{a} \\
u\left(x_{i}\right) / x_{i}\end{array}$ \\
\hline$m_{\mathrm{h}}$ & $u\left(m_{\mathrm{h}}\right)$ & $\begin{array}{l}\text { Weighed } \\
\text { mass of the } \\
\text { tea sample }\end{array}$ & $\begin{array}{c}1,0223 \\
* 10^{-3} \mathrm{~kg}\end{array}$ & $1,3^{*} 10^{-6} \mathrm{~kg}$ & 0,0013 \\
\hline$V_{\mathrm{E}}$ & $u\left(V_{E}\right)$ & $\begin{array}{l}\text { Final volume } \\
\text { of the sample } \\
\text { extract }\end{array}$ & $\begin{array}{c}0,3923 \\
\mathrm{~mL}\end{array}$ & $0,0012 \mathrm{~mL}$ & 0,0030 \\
\hline$c_{\mathrm{a}}$ & $u\left(c_{\mathrm{a}}\right)$ & $\begin{array}{c}\text { Analyte } \\
\text { concentration } \\
\text { determined by } \\
\text { GC-MS }\end{array}$ & $\begin{array}{r}0,497 \\
\mu \mathrm{g} / \mathrm{mL}\end{array}$ & $\begin{array}{c}0,024 \\
\mu \mathrm{g} / \mathrm{mL}\end{array}$ & 0,049 \\
\hline$R$ & $u(R)$ & Recovery & 0,46 & 0,02 & 0,04 \\
\hline$f_{\mathrm{h}}$ & $u\left(f_{h}\right)$ & $\begin{array}{c}\text { Moisture } \\
\text { content }\end{array}$ & 0,916 & 0,003 & 0,004 \\
\hline \multicolumn{6}{|c|}{$\chi_{a}=448 \mu \mathrm{g} / \mathrm{kg}$} \\
\hline \multicolumn{6}{|c|}{$u_{\mathrm{c}}\left(\chi_{a}\right)=28 \mu \mathrm{g} / \mathrm{kg}$} \\
\hline
\end{tabular}

In Fig. 2, all the contributions to the uncertainty of $\chi_{\mathrm{a}}$ are shown in a cause-effect diagram.

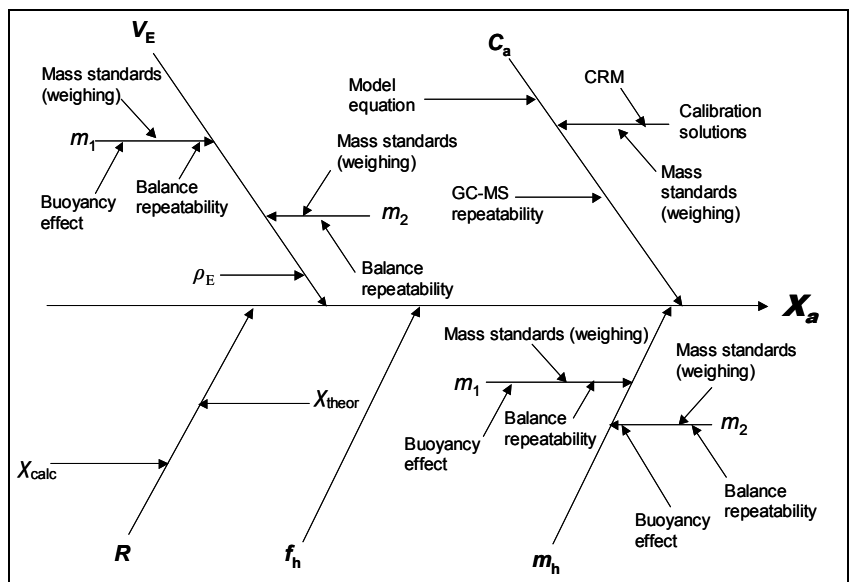

Figure 2. Cause-effect diagram for the uncertainty of $\chi \mathrm{a}$.

\subsection{Conclusions}

The described method represent an example of metrological traceability establishment for the analysis of organic micropollutants in complex matrices. This method allowed to obtain good results for endosulfan sulfate quantification, while $\beta$-endosulfan showed some stability problems during the quantification process, which are under investigation.

\section{Electrolytic Conductivity}

\subsection{Introduction}

The measurement of electrolytic conductivity is a functional analytical tool applied in various fields of science and technology. Although it is a non-specific sum parameter, conductivity measurements yield an estimate of the total concentration of ionized substances in a liquid sample. Under given conditions, conductivity is a useful and accessible quantitative measurement of purity, replacing laborious and expensive chemical analyses. Important applications are in water purity assessment, in pharmaceuticals, drinking water, the food industry, health care, and environmental monitoring.

However, just like any other measurement, conductivity values can be considered reliable only if they are traceable to the International System of Units (SI) [4]. The Istituto Nazionale di Ricerca Metrologica (INRiM) has developed and maintains a metrological reference for electrolytic conductivity measurements in the range from $50 \mu \mathrm{S} / \mathrm{cm}$ to $5 \mathrm{~S} / \mathrm{m}$ with an associated relative uncertainty better than $0,7 \%$ at a temperature of $25{ }^{\circ} \mathrm{C}$. Recently INRiM , in order to answer the needs of industrial and the clinical sectors, has undertaken activities to extend the traceability of electrolytic conductivity measurements to pure water values $(\leq 10 \mu \mathrm{S} / \mathrm{cm})$.

The measurement system under development allows traceable measurements of low conductivity values and, at the same time, secondary cell calibrations. In both cases, significant difficulties arise from (i) solution contamination by air carbon dioxide $\left(\mathrm{CO}_{2}\right)$ and other 
contaminants from the glass walls, (ii) temperature variations and (iii) parasitic phenomena which affect resistance measurements at low frequencies.

\subsection{Fundamentals}

The electrolytic conductivity, $\kappa(\mathrm{S} / \mathrm{m})$, defined as the ratio of the ionic current density to the applied electric field, is the fundamental measurement of the transport of charge through an electrolyte. In practice, $\mathrm{k}$ is evaluated from a measurement of the solution resistance, $R_{\mathrm{s}}(\Omega)$, of a homogeneous, isotropic electrolyte placed between electrodes in a conductivity cell, when the geometric constant $C_{\text {cell }}\left(\mathrm{m}^{-1}\right)$ is exactly known. The fundamental relationship between $\kappa, R_{\mathrm{s}}$ and $C_{\text {cell }}$ is given by equation 3:

$$
\kappa=\frac{C_{\text {cell }}}{R_{s}}
$$

In a cell with parallel, planar electrodes of area $A$, spaced a distance $l$ apart, in which fringe effects are absent and in which the electric field between the electrodes is strictly homogeneous, $C_{\text {cell }}$ equals $l=A$. Cells that are specially developed to maximize homogeneity of the electrical field and for which $C_{\text {cell }}$ is obtained from physical measurements of the relevant geometric quantities, are referred as primary cells.

Then, when $C_{\text {cell }}$ is defined through physical dimensions and the observed value of $R$ is traceable to the SI, the conductivity of an electrolytic sample can be determined in absolute terms because the measurement is carried out with a primary method.

\subsection{Measurement of $\kappa$}

The determination of $\kappa$ of a solution, is usually performed considering impedance measurements using $\mathrm{AC}$, that reduces polarization phenomena at the electrodes.

The resistance, i.e. the real part of the impedance, is measured as a function of frequency. At low frequencies, the resistance increases because of the double layer, while at high frequencies the resistance decreases because of capacitive effects. The resistance, characteristic of the solution bulk, is located at a frequency $f^{*}$, where the modulus of the reactance, i.e. the imaginary part of the impedance, has a minimum.

\subsection{Measurement of low conductivity solutions}

A major application of electrolytic conductivity measurements is related to the evaluation of the ionic purity of water. To ensure the validity of the measured $\kappa$, the sample must be rigidly excluded from exposure to atmospheric carbon dioxide $\left(\mathrm{CO}_{2}\right)$.

Equilibrium with atmospheric $\mathrm{CO}_{2}$ raises the $\kappa$ of a sample of pure water at $25{ }^{\circ} \mathrm{C}$ from $0,055 \mu \mathrm{S} / \mathrm{cm}$ to 1,1 $\mu \mathrm{S} / \mathrm{cm}[5]$.

In addition, electrolytic conductivity is strongly influenced by temperature, especially for high purity water. A solution with conductivity greater than 50 $\mu \mathrm{S} / \mathrm{cm}$, indeed, has a temperature coefficient of approximately $2 \% /{ }^{\circ} \mathrm{C}$ at $25^{\circ} \mathrm{C}$. For high purity water, the conductivity temperature coefficient is approximately $5 \% /{ }^{\circ} \mathrm{C}[6]$. In order to establish the traceability of low conductivity measurements, after the development of a suitable secondary cell [7], a new primary cell was designed and built. In particular, to minimize solution contamination, both from air $\mathrm{CO}_{2}$ and other contaminants, a glass system with flowing solution and argon buffer gas was developed [8, §7.21., 10.4].

\subsection{Experimental set-up}

In this section, the new cells, the glass closed circuit and the experimental set-up for the measurements of low conductivity flowing solutions are described.

The primary cell The new primary cell (Figure 3) for electrolytic conductivity measurements of pure-water was designed at INRiM and developed by the glass blowers Disa Raffaele e F.lli. It is composed of two Pyrex ${ }^{\circledR}$ glass halfcells and a removable hollow cylindrical central section. Each half-cell contains a smooth round planar platinum electrode (thickness $0: 5 \mathrm{~mm}$, diameter $20 \mathrm{~mm}$ ), which guarantees chemical inertness and minimizes the permeability to impurities [6]. These electrodes, embedded into a glass bar, are removable to allow thorough cleaning. Two platinum wires, spot-welded to the back of the electrodes, connect the electrodes to two coaxial connectors. Each half-cell is equipped with two pipes: a filling pipe with an SVL $®$ cap to avoid solution contamination and a valve pipe to allow solution flow.

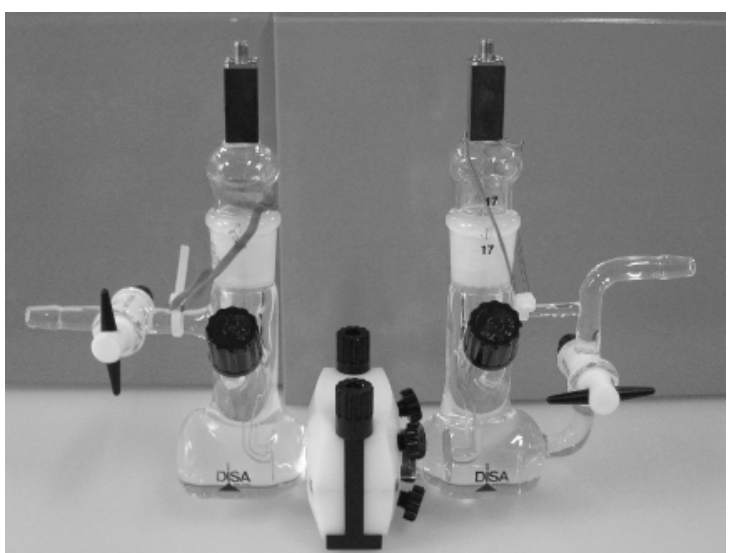

Figure 3. Photograph of the new primary cell developed at INRiM

The removable central section is made of Pyrex ${ }^{\circledR}$ glass. It has a nominal length $l \approx 10 \mathrm{~mm}$ and an inner hole with a nominal diameter $d \approx 15 \mathrm{~mm}$. A special quick closure system in Delrin ${ }^{\circledR}$ (polyoxymethylene) homogeneously distributes mechanical stresses around the glass flanges. Moreover, it tightens the glass flanges preventing lateral and rotational movements of the two half-cells. The cell was designed with a volume of about $160 \mathrm{~mL}$ in order to have a good thermal capacity. 
The secondary cell The secondary cell developed at INRiM has a fixed geometry. It is made of a single Pyrex ${ }^{\circledR}$ glass chamber, holding two platinum parallel and overlooking electrodes. In addition, it has two pipelines that allow the solution flow [7]. The geometric constant of this secondary cell was estimated by calibrating it against the primary cell. This approach uses a primary calibrant (a solution) to determine $C_{\text {cell }}$ for the secondary cell. $\kappa$ of the transfer standard solution is determined using the primary cell, then, the same solution is used to determine $C_{\text {cell }}$ of the secondary cell. The calibrated secondary cell is then used for the characterization of certified reference materials (CRM) and for the calibration of commercial conductivity meters.

Experimental apparatus For the measurement of low electrolytic conductivity values, the primary cell is included in a Pyrex ${ }^{\circledR}$ glass closed circuit in order to have a flowing solution, as shown in figure 4 .

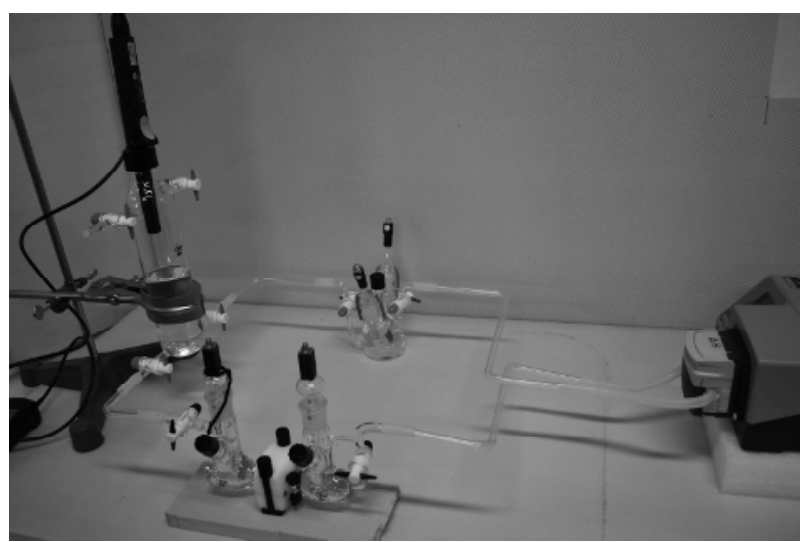

Figure 4: Photograph of the glass closed circuit developed at INRiM

The solution flow $(50 \mathrm{~mL} / \mathrm{min})$ is ensured by a peristaltic pump (Watson-Marlow Bredel Sci- Q 323). The circuit also contains the secondary cell (previously described) and an expansion chamber. The expansion chamber is a Pyrex ${ }^{\circledR}$ glass cylindrical flask equipped with two bottom pipelines, to allow the solution flow, and two top ones to inject an inert gas. This device is partially filled with the solution to be measured and partially with argon gas to avoid solution contamination with air $\mathrm{CO}_{2}$ and to damp possible fast rises of the flow pressure. A commercial $\mathrm{CO}_{2}$ probe (Testo 535) is inserted into the input pipeline of the expansion chamber to monitor the $\mathrm{CO}_{2}$ presence during the measurement period. Silicone joints (Masterflex Biopharm Plus) are used to connect pipelines and other glass components. This material was chosen because of its high inertness, so that solution contamination could be kept to a minimum.

To obtain conductivity values at a reference temperature of $25^{\circ} \mathrm{C}$, the circuit is placed into a thermostatic air bath (Branca Ideal Air). A calibrated Pt100 platinum resistance thermometer is placed in contact with the cell wall and connected to a digital multimeter (Agilent Technologies 3458A) to monitor the solution temperature. An LCR meter (Agilent Technologies E4980A) is employed to perform accurate impedance measurements of the electrolytic solutions.
The applied excitation is a sine wave with an rms value of $0,5 \mathrm{~V}$ : the use of low voltage values avoids phenomena of ion discharge at the electrode surfaces. The electrolytic conductivity of sample solutions is investigated in the 20 $\mathrm{Hz}-2 \mathrm{MHz}$ frequency range. A switch unit (HP3235) is employed to switch the LCR meter from the primary to the secondary cells. Data acquisition is managed by software developed at INRiM in LabWindows/CVI.

\subsection{Measurement method}

Using the primary cell, the electrolytic conductivity is determined by a differential resistance measurement. Thus, the measurement is divided into two distinct phases: in the first phase the primary cell is assembled with the central section, while in the second phase it is assembled without the central section and $\kappa$ of the aqueous solution is estimated by the following equation:

$$
\kappa=\frac{C_{\text {cell }}}{\left(\bar{R}_{W}-\bar{R}_{N}\right)}+\delta \kappa_{W}+\delta \kappa_{N}
$$

$\bar{R}_{W}$ and $\bar{R}_{N}$ are the averages of $\mathrm{n}$ resistance measurements carried out on the cell with (W) and without $(\mathrm{N})$ the central section and corrected for the temperature difference $T_{\mathrm{j}}-T_{\text {ref, }}$, where $T_{\mathrm{j}}$ is the air bath temperature during the $\mathrm{jth}$ measurement. The correction is given by $R_{\mathrm{j}}^{\mathrm{C}}=R_{\mathrm{j}}\left[1+\alpha_{\mathrm{T}}\left(T_{\mathrm{j}}-T_{\mathrm{ref}}\right)\right]$, where $\alpha_{\mathrm{T}}$ is the temperature coefficient of the solution conductivity [6]. Moreover, in equation 4 there are two additional terms, $\delta \kappa_{\mathrm{W}}$ and $\delta \kappa_{\mathrm{N}}$, which take into account the conductivity drift, considering the two configurations, due to solution contamination.

\subsection{Results}

In order to verify the potentialities of the closed circuit, preliminary measurements on a flowing solution of 50 $\mu \mathrm{S} / \mathrm{cm}$ were carried out and a comparison of the stability of a system with flowing solution against that of one without the circuit and with a static solution was performed. The estimated conductivity value was 53,50 $\mu \mathrm{S} / \mathrm{cm}$; the combined standard uncertainty was $9,3 \times 10^{-2}$ $\mu \mathrm{S} / \mathrm{cm}$ and the expanded uncertainty with $\mathrm{k}=2$ was 0,19 $\mu \mathrm{S} / \mathrm{cm}$. The repeatability of the set-up with flowing solution and inert gas was compared with that under static conditions without inert gas. The new set-up achieved a repeatability of about $20 \Omega$ which compares favourably with the static set-up which achieved a repeatability of $52 \Omega$. Using this new experimental setup, additional measurements on lower conductivity solutions $(\leq 10 \mu \mathrm{S} / \mathrm{cm})$ are now in progress. With few changes, calibration tests using a flowing solution will be possible.

\subsection{Conclusion}

A new primary and secondary cells for very low electrolytic conductivity measurements were designed and tested at INRiM . A measurement system with flowing solution was developed to reduce $\mathrm{CO}_{2}$ 
contamination at low conductivity values. Moreover, in this system, the secondary cell is inserted in series with the primary one for in-line calibration.

The old system with static solution and the new one were compared with solutions with low conductivity values $(50 \mu \mathrm{S} / \mathrm{cm})$. The new system showed a reduced drift and a better repeatability: the expanded uncertainty was better than that obtained with a static solution. Future work will be mainly devoted to improve the experimental apparatus: (i) build a new central section with a more accurate cylindrical hole; (ii) substitute the peristaltic pump with a magnetic one; (iii) reduce the number of silicone joints used to connect the glass pipes and the cells and (iv) improve argon monitoring to prevent gas leakage in the system in order to reduce further solution contamination. These arrangements will allow to obtain a system for conductivity measurements of ultrapure water with a target uncertainty lower than $1 \%$.

\section{References}

[1] www.unep.org.

[2] J. Weber, C. J. Halsall, D. Muir, C. Teixeira, J. Small, K. Solomon, M. Hermanson, H. Hung, T. Bidleman, Sci.

Tot. Env., 408, 2966- 2984, (2010)

[3] M. Plassa, M. Mosca, M. Sega, Proceedings of the 16th IMEKO TC3/APMF'98 International Conference on Force, Mass and Torque Measurements (Taejong Korea), 183-191, (1998)

[4] P. Spitzer, B. Rossi, Y. Gaignet, S. Mabic, U. Sudmeier, Accred. Qual. Assur., 10, 3, 78-81, (2005)

[5] K. W. Pratt, W.F. Koch, Y.C. Wu, P.A. Berezansky, (IUPAC Technical Report), Pure Appl. Chem., 73, 11, 1784-1793, (2001)

[6] ASTM Designation, "Standard test method for electrical conductivity and resistivity of water", ASTM D, pp. 1125-1195, (2009)

[7] C. Boveri, F. Durbiano, D. Serazio, Proceedings of the 19th IMEKO World Congress Fundamental and Applied Metrology (Portugal), 2619-2623, (2009) [8] ASTM Designation, "Standard test method for electrical conductivity and resistivity of a flowing high purity water sample", ASTM D, pp. 5391-5399, (2009)

[9] European Co-operation for Accreditation,

"Expression of the uncertainty of measurement in calibration", Publication EA 4/02, (1999)

[10] JCGM, "Evaluation of Measurement Data - Guide to the Expression of Uncertainty in Measurement - $1^{\text {st }}$ edn", JCGM 100:2008, (2008) 